\title{
Modelling the Operating Speed in Segments of Two-Lane Highways from Probe Vehicle Data: A Stochastic Frontier Approach
}

\author{
António Lobo (iD, Marco Amorim, Carlos Rodrigues, and António Couto \\ Research Centre for Territory, Transports and Environment, Faculty of Engineering, University of Porto, Rua Dr. Roberto Frias, \\ $s / n, 4200-465$ Porto, Portugal
}

Correspondence should be addressed to António Lobo; lobo@fe.up.pt

Received 15 November 2017; Revised 5 March 2018; Accepted 13 March 2018; Published 18 April 2018

Academic Editor: Abdelaziz Bensrhair

Copyright @ 2018 António Lobo et al. This is an open access article distributed under the Creative Commons Attribution License, which permits unrestricted use, distribution, and reproduction in any medium, provided the original work is properly cited.

\begin{abstract}
Most of the existing operating speed statistical models are applicable to individual design elements, particularly horizontal curves and tangents. A segment approach to operating speed has rarely been followed, with a few exceptions mainly related to the performance assessment of urban and freeway corridors, or design consistency studies using speed profiles built from successive design elements. This study introduces a new model to predict operating speeds in segments of two-lane highways. The maximum operating speed is given by a stochastic frontier function of the average daily traffic and road geometrics; the asymmetric disturbance accounts for the diversity in drivers' behaviour and vehicle characteristics, allowing estimating any percentile speed. The model was calibrated using probe vehicle data from noncongested roads. The accuracy of the average daily traffic in representing the actual driving conditions was further validated using simultaneous speed-traffic measurements. The new model aims to assist practitioners in the evaluation of design consistency from a macroscopic perspective since the early stages of road planning and design, as well as to support the definition of speed limits at new or existing infrastructures.
\end{abstract}

\section{Introduction}

Increasing efforts to understand and predict operating speeds in roadway facilities have been developed across the past decades, following the tendency of researchers and practitioners to use the operating speed as an instrument to define road geometrics and promote design consistency. The great majority of the existing models are focused on the purely geometric effects on driving speeds, exploring the relations between geometric parameters and the speeds practiced under free-flow conditions in individual design elements, especially in horizontal curves or tangents. Such models are commonly known as spot speed models, of which the Transportation Research Circular E-C151 [1] provides a comprehensive review.

The operating speed in a road segment, defined as a continuous sequence of design elements composing a stretch of road, has been much less studied, despite the relevance that segment speed estimations based on roadway characteristics may present to the evaluation of road design and operations. The use of segment speed prediction models since the early stages of road design allows practitioners to verify, for each design iteration, if the differences between the selected design speed and the expected operating speed lie within acceptable limits. A sensitivity analysis on such differences could shed light on the variables most influencing design consistency, thus providing an insight into the most effective actions to control it in the following iterations of the design process, with the ultimate goal of obtaining a satisfactory final solution. This procedure is in line with the suggestions made by Fitzpatrick et al. [2] regarding the use of alignment indices to evaluate design consistency from a macroscopic perspective. Nevertheless, to improve safety performance, Fitzpatrick et al. [2] advocate that such analysis should be complemented by a conventional speed profile approach based on the evaluation of speed reductions between successive design elements. 
Segment speed models may also play an important role in the definition of speed limits in planned roads or in the adjustment of the existing ones. Speed limits are legislated by road functional class, but road designers and managers may establish speed zones with different limits if the statutory limits do not comply with the specific road or traffic conditions. The most common approach consists in setting the speed limit near the 85th-percentile speed (V85) at representative locations of the proposed zone [3]. The use of segment speed models avoids a subjective choice of representative sites by considering the aggregated characteristics of entire zone. Additionally, the model can be used in alternative methods to define speed limits, such as the expert judgment [4-6], assisting experts in the proposal of adequate speed limits.

Finally, practitioners may use segment speed estimations in the evaluation and monitoring of existing roads when real-world speed data is not available. The evaluation of travel times, costs, and emissions, the identification of safety hazards, and the information of road users are examples of applications that frequently rely on the knowledge of operating speeds and that may lead practitioners to take measures aimed at improving the system's performance and efficiency.

To address the lack of operating speed research in road segments, this study presents a new model for twolane highways. The main objective is to deliver a novel segment speed tool, capable of predicting any user-specified percentile, to assist practitioners in the design, safety analysis, and performance evaluation of new and existing roadway infrastructures, focusing on rural roads where speed monitoring equipment is rarely available. The segment speed model builds on the authors' previous research on spot speed modelling, retaining the operating speed frontier model (OSFM) formulation that allows percentile speed estimation $[7,8]$. In the new model, the geometric and traffic characteristics are represented by a frontier function, while an asymmetric disturbance term accounts for nonquantified factors related to drivers' behaviour and vehicle technology. Segment speeds were assessed from probe vehicle data, geometric characteristics were assessed from both probe vehicle data and in situ measurements, and traffic effects were evaluated through the annual average daily traffic.

The remainder of the paper is structured as follows. First, a background on the most relevant achievements contained in the literature concerning the study of segment speeds is provided. The data description details the speed and road geometry data collection using probe vehicles and the additional data requirements in terms of geometric and traffic characteristics. Then, the model description presents the OSFM formulation and the methods used to derive the new segment speed model from a previous spot speed model presented by the authors [8]. The model estimation introduces the segment speed model for two-lane highways, calibrated for Portuguese conditions. The paper proceeds with the comparison between the OSFM and a speed profile method, and with the model validation using simultaneous speed and traffic data from road segments beyond the calibration sample. The main conclusions of this study are presented in the final section.

\section{Background}

The array of segment speed models presented in the literature is much smaller than the comprehensive set of spot speed models developed to date [1]. The main cause of the lack of development of segment speed studies is probably related to the need for more time and significantly more resources to perform data collection.

The traditional techniques to assess segment speeds are the stationary observer method and the moving observer method [9]. In the stationary observer method, an observer is placed at each end of the survey segment recording each vehicle's passing time and license plate, in order to calculate the time spent to traverse the segment. In the moving observer method, an observer in a test vehicle chases randomly chosen vehicles across the segment, recording the corresponding travel times. The underlying speed concept in both methods is the space mean speed, represented by the harmonic mean of speeds over a length of roadway, which can be derived from the average travel time of the observed vehicles.

In freeways and urban roads, researchers have taken advantage of available technology devices to reducing human intervention in data collection. Dion and Rakha [10], Li et al. [11], and Tam and Lam [12] applied the principle of the stationary observer by using automatic vehicle identification (AVI) data from toll collection points to compute travel time and speed. Jenelius and Koutsopoulos [13] and Wang et al. [14] applied the principle of the moving observer to measure travel times and speeds in urban links using floating car data (FCD) from probe systems installed in taxi fleets. Rakha and Zhang [15], Soriguera and Robusté [16], and Martínez-Díaz and Pérez [17] derived space mean speeds and corresponding confidence intervals from time mean speeds in freeways, taking advantage of existing loop detectors. While these studies have predominantly followed a statistical approach, authors such as Park et al. [18], van Lint et al. [19], and Tang et al. [20] applied neural networks to forecast speed and travel time using data from fixed devices installed in urban freeway corridors, highlighting the advantages of these methods to provide short-term predictions in periods of congestion.

In relation to two-lane highways, only a few design guides propose methods to define operating speeds over a length of roadway. In the UK, both operating and design speeds are established on the basis of geometric variables, particularly the bendiness and mean visibility observed on a minimum $2 \mathrm{~km}$ long segment [21]. In Germany, the operating speed is determined by the curvature change rate and pavement width [22]. Other official guidelines propose different design speeds according to the road functional classification and provide methods to ensure design consistency by limiting speed differences between successive design elements. This is the case of the US reference manual A Policy on Geometric Design of Highways and Streets [23] and the Portuguese guidelines [24].

The Highway Capacity Manual (HCM) [25], which is regarded by practitioners worldwide as a standard reference on capacity and level-of-service procedures, also uses a segment approach to estimate the free-flow speed (FFS). First, a base free-flow speed (BFFS) based on the design speed, posted speed limit, or speeds observed in similar facilities 


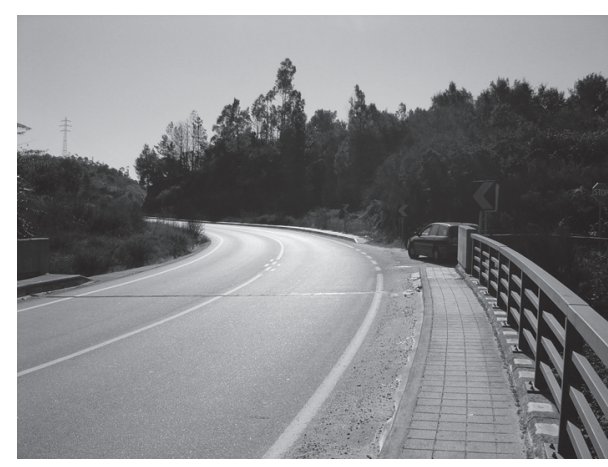

(a)

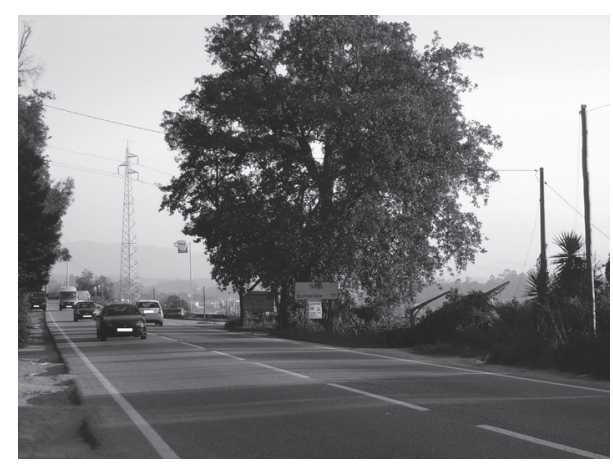

(b)

Figure 1: Examples of the considered roads: N 101 (a) and N 206 (b).

must be adopted. Then, the FFS is estimated by reducing the BFFS for the effects of the cross-section width and density of access points. The HCM justifies that no further guidance on estimating the BFFS is provided because of the diversity of local conditions that play a major role in drivers' speed choice. However, it is possible to conclude that the BFFS is the speed observed at roads with a similar functional classification and design standards, with no access points, and lane and shoulder widths equal to or greater than $3.6 \mathrm{~m}$ and $1.8 \mathrm{~m}$, respectively, since the proposed corrections to the BFFS are only applicable for higher densities of access points and smaller cross-section widths. The HCM suggests the use of this method only when speed measurements are not possible, for example, at the planning and design stages of a new road, but speed estimation methods for other facilities are still required if the BFFS is established by comparison with similar roads.

In sum, the academic community has developed some methods to estimate segment speeds in freeways and small urban links, using data from existing monitoring equipment to simplify data collection. In turn, reference manuals provide some guidance to define design and/or operating speeds in roads of different functional classifications, including in twolane highways, but are relatively coy about explaining the underlying methods and assumptions. Therefore, the segment speed model developed in this study aims at presenting a significant contribution to this branch of knowledge.

\section{Data Description}

The new segment speed model was calibrated using speed, traffic, and geometrics data from nine road segments contained in three two-lane highways in Portugal: N 14, N 101, and $\mathrm{N} 206$. In these roads, design speeds range from 40 to $60 \mathrm{~km} / \mathrm{h}$, junctions are usually at-grade, and direct access to marginal properties is allowed. Additionally, roadside occupation has a great influence on the density of intersections and lateral clearance. The curve radius and lane width are sometimes smaller than the minimum values proposed by the guidelines [24]. The case-study roads were selected to reflect the diversity of characteristics among the National Roads, a category that represents the vast majority of the country's highway network. Roads of higher functional classifications, that is, Principal and Complementary Itineraries, were not included in the present study due to data availability issues.

Each calibration segment is between 2 and $4 \mathrm{~km}$ long. The total extension of analysed roadway is of $23.5 \mathrm{~km}$. The segments are located outside the urban areas, crossing either completely rural settings or zones with scattered buildings (Figure 1). The pavement is well maintained and the markings are clearly visible. The topographic features are consistent with the classification of level or rolling terrain [25]. The traffic is characterized by a noncongested flow, except for the possible occurrence of isolated incidents. Segments containing signalized intersections or roundabouts were excluded from this analysis, ensuring that all the vehicles traversing the calibration segments have right-of-way.

Speed data was derived from probe vehicle data provided by a company specialized in digital mapping and applications. This company runs numerous probe information devices, installed on board of professional and private vehicles, which feed a real-time traffic information system available to media partners and GPS applications. A high-rate GPS system $(>1 \mathrm{~Hz})$ provides an accurate representation of each probe vehicle's trajectory, allowing identifying the vehicles that have traversed each calibration segment. The space mean speed of each vehicle, consisting of the model's dependent variable, was calculated from the travel time given by the difference between GPS timestamps corresponding to the beginning and the end of the segment. To ensure a homogenous modelling sample, 75 vehicles observed in each segment in both directions were considered, corresponding to the amount of probe vehicles at the calibration segment with less recorded observations. In total, 675 observations were used for model estimation.

The independent variables included in the segment speed model are based on both geometric and traffic characteristics. The geometric design is characterized by the horizontal alignment, cross-section width, and density of intersections, which is in line with previous research on the speed effects produced by variables measured over a length of roadway $[8$, $21,22,25,26]$. The horizontal alignment of the calibration segments was reproduced in a CAD software, using probe data collected by the instrumented vehicle of the Traffic Analysis Laboratory of the Faculty of Engineering of the University of 
TABLE 1: General data on calibration segments.

\begin{tabular}{|c|c|c|c|c|}
\hline Variable description & Mean & Standard deviation & Minimum & Maximum \\
\hline Space mean speed $(\mathrm{km} / \mathrm{h})$ & 49.2 & 9.2 & 21.0 & 80.0 \\
\hline Bendiness $(B)[$ degrees $/ \mathrm{km}]$ & 306.7 & 207.0 & 39.0 & 682.3 \\
\hline One-direction paved width (PW) $[\mathrm{m}]$ & 4.2 & 0.5 & 3.4 & 5.4 \\
\hline Extra lateral clearance (ELC) $[\mathrm{m}]$ & 1.2 & 0.4 & 0.7 & 2.2 \\
\hline Density of intersections (DI) [No./km] & 4.0 & 1.8 & 0.5 & 7.0 \\
\hline Annual average daily traffic (AADT) [vehicles/day] & 8,736 & 5,545 & 1,750 & 18,135 \\
\hline
\end{tabular}

Porto. A similar reproduction of the vertical alignment was not possible due to unreliable altimetry data. The Highway Capacity Manual [25] suggests that relevant speed reductions may occur in segments with grades exceeding 3\% for more than $1 \mathrm{~km}$. However, this situation does not occur in the calibration segments, although steeper grades may locally be present. Thus, the lack of variables characterizing the vertical alignment is not expected to represent a relevant omission in the model.

Contrary to spot speed models usually dealing with freeflow vehicles, segment speed models should account for the traffic effects. First, the evaluation of the operational performance of a roadway infrastructure using the latter models will be more realistic if traffic conditions are considered. Second, independently of the method used to collect segment speed data, it is difficult to guarantee that sample vehicles traverse the entire segment under free-flow conditions; hence speed observations may have been affected by the presence of other vehicles on the road.

Specifically, the geometric and traffic characteristics included in the model were the bendiness $(B)$, the onedirection paved width (PW) and its standard deviation (SDPW), the extra lateral clearance (ELC), the density of intersections (DI), and the annual average daily traffic (AADT). $B$ corresponds to the sum of the deflection angles of the horizontal alignment per kilometre. PW represents the mean value of the lane and right shoulder combined width. The mean was calculated from measurements taken at a set of locations within the segment. The spacing between measurement spots is approximately $500 \mathrm{~m}$. PW experiments frequent variations according to the marginal land use and topography across Portuguese National Roads, especially concerning the shoulder width. Therefore, SDPW was included in the model to account for the effects of such diversity within a road segment. ELC represents the distance between the right shoulder external limit and any fixed object at the roadside. This variable was defined by the mean value corresponding to the same sites where PW was measured. DI corresponds to the number of intersections or interchanges per kilometre. An aggregated daily traffic measure, AADT, was considered because the time of day at which probe vehicles were recorded is unknown. Then, AADT was used as a proxy for the momentary traffic conditions faced by the test vehicles. Traffic data was either provided by a previous accident modelling study conducted at the same roads by Costa [27] or estimated from in situ measurements using automatic traffic counters (VIACOUNT II). The use of daily traffic measures as speed predictors is not new among the literature, being present in studies such as Lamm and Choueiri [28], Jessen et al. [29], and Schurr et al. [30].

Because all the variables encompass a spatial dimension, reflecting either a value per unit of distance or an average value representative of the entire segment, the segment length was not included in the analysis. The minimum length of $2 \mathrm{~km}$ required to select a calibration segment ensures a reasonable distance to assess space mean speeds, as well as the necessary differentiation from spot speed studies. Table 1 presents the general description of the data used to estimate the segment speed model.

\section{Model Description}

The OSFM used in this research was introduced in previous spot speed studies by the authors $[7,8]$ to address some limitations of the widespread percentile-specific models [1]. Traditionally, the calibration of speed models is made by applying a linear regression between speed factors and a specific percentile speed obtained by the aggregation of values collected in situ. Besides the obvious disadvantage of supporting the estimation of only one percentile speed, speed data aggregation in conventional models causes a loss of information that reduces the total variability and the nature of the variability associated with the regression function. Consequently, the estimated effects of the independent variables may be biased [31]. Therefore, modelling the entire speed distribution may contribute to rectifying this problem.

The literature contains only two other models supporting a speed distribution that can be used to estimate any percentile spot speed. Medina and Tarko [32] employed an ordinary least squares method to estimate a customized regression equation for any intended percentile. The resulting models represent a linear combinations of factors associated with the mean and the standard deviation of the speed distribution. Hewson [33] generalized this approach by using quantile regression.

The OSFM [7, 8], albeit using the entire speed distribution, follows a completely different approach based on stochastic frontier models from the econometric analysis [34, 35]. The OSFM is formed by a deterministic speed frontier function and two disturbance terms. The speed frontier is given by an exponential function of a set of independent variables characterizing the most relevant road conditions, being estimated by the maximum likelihood method using the total number of speed observations. The exponential form allows for the interaction between the order of magnitude of speeds and the impacts of the independent variables. The 
values upon the speed frontier represent the upper limit of the operating speed estimated for each combination of independent variables; hence, the speed frontier is dubbed the maximum operating speed $(V \max )$. In other words, $V \max$ reflects the speed pattern of the fastest drivers and varies according to the conditions presented by the road. $V \max$ results from the statistical processing of operating speeds collected under good weather and pavement conditions; thus it should not be mistaken for speed concepts representing safety-critical situations for the vehicle's dynamic equilibrium.

The segment speed model is based on the authors' latest spot speed model for horizontal curves and tangents of Portuguese National Roads, introduced in Lobo et al. [8]. In this spot speed model, $V \max$ is composed of variables characterizing the local conditions, namely, on-site geometric and visibility parameters, and variables reflecting the recent driving experience, that is, geometric and roadside interference parameters measured over the preceding segment. The general formulation presented by Lobo et al. [8] that supports the prediction of $V \max$ at an individual design element is given by

$$
V \max _{e}=\exp \left(\beta_{0}+\sum_{k=1}^{n_{k}} \beta_{k} \ln X_{k}+\sum_{m=1}^{n_{m}} \beta_{m} \ln X_{m}\right),
$$

where $V \max _{e}$ is the maximum operating speed at a given design element, $X_{k}$ is on-site characteristic $k, X_{m}$ is segment characteristic $m$, and $\beta$ are regression coefficients.

The new segment speed model is developed from the previous spot speed model, not only because both models adopt an OSFM formulation, but also because the aggregated effect produced by the segment characteristics on the operating speed is assumed identical in both models except for a scale factor. In other words, the segment effects included in the spot speed model translate into the segment speed model through the calibration of a single coefficient affecting all those variables. The variable SC, used as a predictor in the segment speed model, combines the segment characteristics considered in the spot speed model, such as SC = $\exp \left(\sum_{m=1}^{n_{m}} \beta_{m} \ln X_{m}\right)$. Additionally, the new model accounts for specific effects affecting segment speeds that were absent from the spot speed model. These effects are related to the presence of traffic and the dispersion of the crosssection width, as previously discussed in this paper. Variables characterizing individual design elements, that is, measured locally instead over a length of roadway, are excluded from the segment speed model. Equation (2) represents the general formulation of the model to estimate $V \max$ at a given road segment.

$$
V \max _{s}=\exp \left(\alpha_{0}+\alpha_{1} \ln \mathrm{SC}+\sum_{q=2}^{n_{q}} \alpha_{q} \ln X_{q}\right)
$$

where $V \max _{s}$ is the maximum operating speed at a given road segment, $\mathrm{SC}$ is the variable representing the aggregated segment characteristics from the spot speed model, $X_{q}$ is segment characteristic $q$ related to traffic or the dispersion of cross-section variables, $\alpha_{0}$ is the regression constant, $\alpha_{1}$ is the regression coefficient or scale factor of variable SC, and $\alpha_{q}$ are regression coefficients of the segment characteristics $q$.

The OSFM considers two disturbance terms: the noise term and the asymmetric disturbance term. The former term supports the stochastic nature of the speed frontier, representing random errors associated with model specification and data collection. It is frequently considered in regression models and assumes a normal distribution. The latter term is an asymmetrically distributed disturbance accounting for the deviations from $V \max$ that are attributed to nonquantified factors, that is, other factors than those represented by the deterministic frontier. Then, the asymmetric disturbance is suitable to represent the diversity of vehicle speeds observed at the same road element or segment, which, in turn, depends on the individual responses of each pair driver-vehicle to similar road conditions. In other words, the asymmetric disturbance in the OSFM accounts for the speed reductions from $V$ max caused by the diversity in drivers' behaviour and vehicle technology. Therefore, the cumulative distribution function of this disturbance allows estimating any desired percentile speed. Similarly to the previous studies $[7,8]$, the asymmetric disturbance adopts an exponential distribution, such as $f(u)=\theta \times \exp (-\theta u)$, where $\theta$ is the rate parameter of the exponential function. The cumulative function of the asymmetric disturbance is given by $F(u)=1-\exp (-\theta u)$, and the inverse transform is $u=(-1 / \theta) \times \ln (1-F)$. Hence, the $p$ th percentile speed at a given road segment can be estimated using

$$
V p_{s}=V \max _{s} \times \exp \left(\frac{1}{\theta} \ln p\right),
$$

where $V p_{s}$ is the $p$ th percentile speed a given road segment and $p$ is the percentile value $(0<p<1)$.

According to Greene [36], the maximum likelihood method is more efficient in processing asymmetric disturbances than the least squares method. Thus, the OSFM is estimated through the maximization of the log-likelihood function shown in

$$
\begin{aligned}
\ln L= & N \ln \theta+\frac{N}{2} \theta^{2} \sigma_{v}^{2}+\theta \sum_{j=1}^{n_{j}} \sum_{i=1}^{n_{i}}\left(v_{i j}-u_{i j}\right) \\
& +\sum_{j=1}^{n_{j}} \sum_{i=1}^{n_{i}} \ln \Phi\left(-\frac{v_{i j}-u_{i j}}{\sigma_{v}}-\theta \sigma_{v}\right),
\end{aligned}
$$

where $L$ is the likelihood function, $N$ is the total number of observations, $\sigma_{v}$ is the standard deviation of the noise term distribution, $v_{i j}$ is the noise term for vehicle $i$ in segment $j$, $u_{i j}$ is the asymmetric disturbance for vehicle $i$ in segment $j$, and $\Phi(\cdot)$ is the standard normal distribution function.

\section{Model Estimation}

The calibration of the segment speed model for National Roads in Portugal was made through a stochastic frontier regression between the space mean speeds of all sample 
TABLE 2: Results of the stochastic frontier regression.

\begin{tabular}{lcc}
\hline Variable & Coefficient & Standard error \\
\hline Constant & 4.846 & $0.197^{\mathrm{a}}$ \\
$\ln \mathrm{SC}$ & 4.462 & $0.437^{\mathrm{a}}$ \\
$\ln \mathrm{SDPW}$ & -0.125 & $0.019^{\mathrm{a}}$ \\
$\ln \mathrm{AADT}$ & -0.064 & $0.019^{\mathrm{a}}$ \\
\hline
\end{tabular}

Note. Log-likelihood $=143.617$; number of observations $=675 ; \sigma_{u}=0.168 ; \sigma_{v}$ $=0.124 ; \theta=5.947 .{ }^{\mathrm{a}}$ Significant at $1 \%$ level.

vehicles and the road geometrics and traffic. The regression is based on

$$
\begin{aligned}
\ln V p_{s}= & \alpha_{0}+\alpha_{1} \ln \mathrm{SC}+\alpha_{2} \ln \mathrm{SDPW}+\alpha_{3} \ln \mathrm{AADT} \\
& +\frac{1}{\theta} \ln p,
\end{aligned}
$$

where SC $=\mathrm{PW}^{0.079} \times \mathrm{ELC}^{0.008} \times B^{-0.027} \times \mathrm{DI}^{-0.036}$.

Equation (5) represents a linearized form of (3) and details the model formulation with the previously described variables. The exponents of the factors composing SC originate from the spot speed model for Portuguese National Roads presented by Lobo et al. [8], being recalibrated in the segment speed model by the scale factor $\alpha_{1}$. To comply with the OSFM formulation, the speed predictors are transformed in log terms, which implies that the model is not applicable when ELC, $B$, DI, or SWPD is null.

The model was estimated through the maximum likelihood method, using the econometric software Limdep [37]. The results are shown in Table 2. These results translate into the final specification for the model to estimate $V \max$ and the $p$ th percentile speed in segments of Portuguese National Roads represented by

$$
\begin{aligned}
& V \max _{s}=\exp (4.846+4.462 \\
& \times \ln \left(\mathrm{PW}^{0.079} \times \mathrm{ELC}^{0.008} \times B^{-0.027} \times \mathrm{DI}^{-0.036}\right) \\
& -0.125 \times \ln \mathrm{SDPW}-0.064 \times \ln \mathrm{AADT}) \\
& V p_{s}=V \max _{s} \times \exp \left(\frac{1}{5.947} \times \ln p\right)
\end{aligned}
$$

with ELC, $B$, DI, and SWPD $>0$.

The coefficients of the individual variables can be interpreted as elasticities due to the model's functional form. The elasticities of PW, ELC, $B$, and DI are given by the original elasticities estimated in the spot speed model multiplied by the scale factor $\alpha_{1}$. The results show that the geometric variables, particularly those related to the cross-section width, represent the most relevant effects affecting operating speeds. An increase in PW while keeping the remaining variables constant produces a positive impact on speed, materialized by an elasticity of 0.352 . The impact of increasing ELC is also positive, albeit with a smaller elasticity of 0.036 . Additionally, the negative coefficient of SDPW reflects that the variation of PW over a road segment has a negative impact on speed. Many Portuguese National Roads are characterized by an inconsistent paved width, mainly attributed to frequently changing shoulder widths according to the marginal land use, which introduces uncertainty in drivers' expectations about the road ahead, leading them to adopt a more cautious speed choice. The speed impacts caused by increases in the density of intersections and bendiness are negative, with elasticities of -0.161 and -0.120 , respectively. The results also show that, as expected, increasing traffic leads to speed reductions. Particularly, the elasticity associated with AADT denotes that a traffic increase of $10 \%$ under a noncongested flow would produce a decrease in the space mean speed of $0.6 \%$.

\section{Model Validation}

The model's capability of supporting accurate speed predictions was evaluated using two different procedures. First, the speed predictions returned by the model for each one of the calibration segments were compared against the results of a speed profile method. Then, the model was validated using simultaneous speed and traffic data collected in two additional road segments.

6.1. Comparison with a Speed Profile Approach. To the best of the authors' knowledge, there is no other segment speed model for two-lane rural highways that was developed outside the scope of road design and capacity manuals. In Portugal, the official guidelines propose a speed profile method to evaluate design consistency over a length of roadway from a safety perspective. Speed is computed element by element, taking into account the vehicle's dynamic equilibrium, to verify if the differences between successive design elements lie within the regulatory limits [24]. Based on this approach, a speed profile approach was used to estimate segment speeds at the nine calibration segments, CS1 to CS9, and compare the results with the OSFM estimations. To comply with the operational nature of this analysis, the dynamic equilibrium equations were replaced by a conventional V85 model previously developed for spot speed prediction in curves and tangents of Portuguese two-lane roads [26]. Then, an approximation of the travel time in each design element was obtained from the estimated V85; the model does not consider speed variations within the design elements. Finally, segment speeds were derived from the sum of travel times of all the design elements composing each segment. Figure 2 presents the observed 85th-percentile segment speeds (V85so) and the corresponding estimations using the OSFM $(\mathrm{V} 85 \mathrm{sm})$ and the speed profile method (V85ssp).

In seven out of nine cases, the differences between the OSFM estimations and the observed values are smaller than $4 \mathrm{~km} / \mathrm{h}$, outperforming the speed profile method. Although the latter method returns better predictions in the remaining two cases, the deviations from the observed values across the test cases are much more inconsistent, which may be related to the nonconsideration of traffic and acceleration/deceleration effects.

The mean absolute deviation (MAD) and the mean squared error (MSE) were calculated for both methods using the set of nine test cases. The OSFM estimations resulted in a MAD of 3.4 and a MSE of 32.1. The speed profile method produced a MAD of 8.4 and a MSE of 128.3. These 


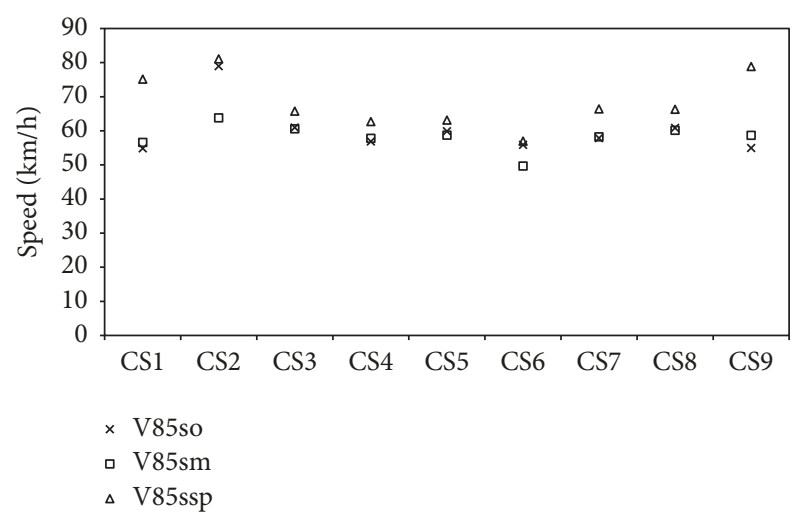

FIgURE 2: Comparison between the OSFM and a speed profile method.

TABLE 3: Geometric and traffic characteristics of the validation segments.

\begin{tabular}{lcc}
\hline Variable & VS1 & VS2 \\
\hline$B($ degrees $/ \mathrm{km})$ & 199.3 & 63.5 \\
PW $(\mathrm{m})$ & 4.4 & 6.6 \\
ELC $(\mathrm{m})$ & 1.4 & 1.0 \\
DI (number/km) & 4.3 & 3.0 \\
AADT (vehicles/day) & 6,193 & 15,290 \\
\hline
\end{tabular}

results show a much better fit of the OSFM compared to the speed profile approach, regarding the estimation of V85. Additionally, the OSFM was used to estimate the percentile speeds between the 1st and the 99th for all the calibration segments. A MAD of 4.5 and a MSE of 33.1 were obtained with this sample, denoting that the model's goodness-of-fit is reasonably stable across different percentiles.

6.2. Validation with Simultaneous Speed and Traffic Data. The segment speed model was validated in two additional road segments, VS1 and VS2, to test if the use of an aggregated traffic measure, AADT, does not affect the model's ability to cope with different traffic situations. In this sense, percentile speeds obtained from the model and from measurements conducted at the two validation segments were compared against each other for equivalent traffic flows. The comparison was made for high and low volume conditions, which were associated with peak and daytime off-peak periods, respectively. The nighttime off-peak period was not analysed because it is assumed that the traffic flow is low enough to ensure free-flow conditions for almost all the vehicles.

Both VS1 and VS2 are $3 \mathrm{~km}$ long and belong to two Portuguese National Roads not included in the modelling sample, respectively, $\mathrm{N} 108$ and $\mathrm{N} 222$. The former road is much older than the latter, which translates into lower standards in terms of horizontal curvature, cross-section width, and roadside interference. The traffic in VS1 is lower than in VS2 because the former is located farther away from a major urban centre. Table 3 contains the characteristics collected for both validation segments.

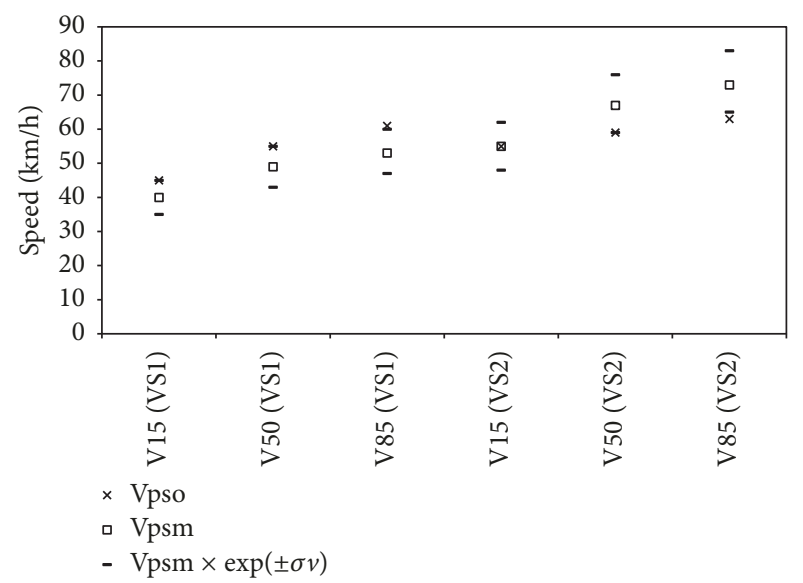

FIGURE 3: Validation results for conditions of high traffic volume.

The procedure started with the implementation of 24hour traffic measurements to characterize both high and low volume traffic conditions at each segment in a labour day. An automatic traffic counter (VIACOUNT II) was placed approximately at the midpoint of each segment. The highest half-hourly traffic volume was observed during the afternoon peak in both segments, specifically between 5:45 and 6:15 PM in VS1 and between 6:00 and 6:30 PM in VS2. The lowest halfhourly volume during the daytime was observed between the morning and the afternoon traffic peaks, from 4:45 to 5:15 PM in VS1 and from 4:15 to 4:45 PM in VS2. The half-hourly basis was selected to contain the use of resources in the following step, conducted at the equivalent time periods of another labour day. In that step, an observer recording the vehicles' license plates and passing times and an automatic traffic counter for simultaneous and reliable traffic measurements were placed at both ends of each validation segment. The license plate recognition allowed deriving segment percentile speeds from in situ observations $\left(V p_{s o}\right)$ for high and low traffic volume conditions. In turn, the simultaneous traffic measurements allowed obtaining segment percentile speed estimations from the model $\left(V p_{s m}\right)$. Because the model uses $\mathrm{AADT}$ as an explanatory variable, the half-hourly traffic characterizing periods of high and low volume were converted to a daily basis by multiplying for 48 . This transformation led to $V p_{s m}$ estimations corresponding to hypothetical situations of constant traffic flow during 24 hours. Although such situations are unrealistic, this procedure ensures that $V p_{s m}$ and $V p_{s o}$ report similar traffic flows.

The comparison between $V p_{s m}$ and $V p_{s o}$ was performed through the 15th, 50th, and 85th percentiles (V15, V50, and V85), which are the most commonly used in speed modelling applications. To better guide this comparison, the random error component was applied to speed estimations in the form of an interval defined around $V p_{s m}$ by the standard deviation of the noise term distribution, such that the limits of the interval are given by $V p_{s m} \times \exp \left( \pm \sigma_{v}\right)$. The results are plotted in Figures 3 and 4, corresponding to the conditions of high and low traffic volumes, respectively.

For the condition of a high traffic volume, the observed percentile speeds lie within the interval defined by the 


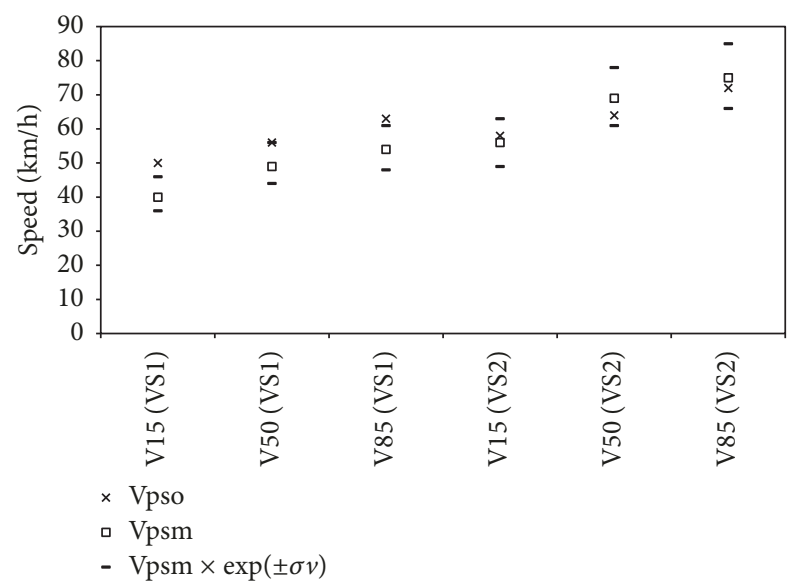

FIGURE 4: Validation results for conditions of low traffic volume.

standard deviation of the noise term in four out of six cases, with the V85 estimations sitting $1 \mathrm{~km} / \mathrm{h}$ above and $2 \mathrm{~km} / \mathrm{h}$ below the interval for VS1 and VS2, respectively. For a low traffic volume, the observed values are also contained in the interval in four out of six cases. The observed V15 and V85 in VS1 surpass the upper limit of the interval by $4 \mathrm{~km} / \mathrm{h}$ and $2 \mathrm{~km} / \mathrm{h}$, respectively.

These results may denote a slight underestimation of the segment percentile speeds by the model in National Roads with lower design standards, with half of the observed values presented for VS1 being placed above the corresponding intervals. The model performs better in the case of VS2, representing a National Road with a higher design speed, with just one of the observed percentile speeds falling below the interval for a small margin. The segment speed model also seems to return more accurate predictions for medium-tosmall percentiles.

However, from a global perspective, the observed percentile speeds are contained by the interval around the estimated values in eight out of twelve cases. In the remaining four cases, speed deviations from the corresponding intervals are, at most, of $4 \mathrm{~km} / \mathrm{h}$. Therefore, the selected speed predictors, including AADT, do not seem to affect the validity of the segment speed model, which has revealed the capability to support reliable speed estimations under different geometric and traffic conditions.

\section{Conclusions}

Speed is one of the most important factors affecting the convenience and efficiency of roadway infrastructures. Drivers' perception of the operating speeds of different routes and, consequently, of travel times and costs strongly influences their route choice. In turn, road designers and managers use the operating speed to evaluate and monitor the performance of infrastructures in terms of safety, traffic flow, and environmental efficiency.

Notwithstanding the notable contributions presented by numerous spot speed models existing in the literature, the evaluation of road performance can be significantly improved through the development of segment speed methods. The estimation of operating speeds over a length of roadway is usually associated with the measurement of the corresponding travel times, which can be very time and resource consuming, especially if no automatic data collection is involved. In practice, such difficulties have been hampering the development of new segment speed prediction tools; most research in this field has been limited to freeway and urban links, taking advantage of data collected by monitoring equipment owned by road authorities and concessionaires [17].

This study delivers the only operating speed model applicable to segments of two-lane highways that was developed to date outside the scope of official guidelines, with the major advantage of supporting any user-specified percentile speed prediction. The model can be particularly useful in road design and safety applications, such as the evaluation of design consistency from an early design stage and the definition of speed zones. Additionally, it can provide speed predictions to assist long-term network management actions in roads without permanent traffic monitoring equipment, a situation that is very common in the case of two-lane rural highways.

The model evolves from the authors' previous research on spot speed modelling, retaining the OSFM formulation presented in Lobo et al. [7, 8]. In this sense, road geometry and traffic characteristics are used to estimate a deterministic speed frontier, representing the maximum operating speed for each combination of explanatory variables. The asymmetric disturbance accounts for nonquantified factors associated with drivers' behaviour and vehicle technology, with the estimation of percentile speeds being made by means of the cumulative distribution function of this disturbance.

The segment speed model was calibrated using the entire speed distribution from a probe vehicle database, observed in nine segments of Portuguese National Roads. Because simultaneous speed and traffic data was not available for the calibration segments, AADT was used to represent traffic conditions. The model estimation results confirm the primary influence of geometric characteristics on the speeds practiced under noncongested traffic conditions; the impact of traffic is much smaller and predictably negative.

The OSFM was compared against a speed profile method with positive results. Additionally, it was validated using license plate recognition in two road segments beyond the calibration sample, each one presenting differentiated geometric and traffic characteristics. This procedure allowed obtaining simultaneous speed and traffic data, which was used to assess percentile speeds from both in situ measurements and model estimations. The model has proved reliable in its predictions, with the observed speed values lying within or sufficiently close to the interval around the estimated values defined by the standard deviation of the noise term distribution. Therefore, this study confirms the adequacy of using probe vehicles to collect speed and road geometry data for speed modelling applications.

It should be noted that the model was calibrated for noncongested National Roads in Portugal. Therefore, it is not valid for congested traffic flow. Additionally, its application to a different regional context should be carefully considered, especially in non-European countries where geometric 
standards may vary significantly. The OSFM formulation is, however, flexible enough to be replicated in other regions with different road conditions. The lack of tools for segment speed prediction in two-lane highways further highlights the contribution of the present study to road performance and safety applications, particularly to the control of design consistency from the early planning stages, but also to the definition of speed limits, the evaluation of travel times, and the recommendation of efficient routes.

\section{Conflicts of Interest}

The authors declare that there are no conflicts of interest regarding the publication of this article.

\section{Acknowledgments}

The authors would like to thank the Portuguese Foundation for Science and Technology for funding this research through the Ph.D. Studentship SFRH/BD/71322/2010 and InfoPortugal, S.A., for providing the probe vehicle data.

\section{References}

[1] Transportation Research Board (TRB), "Modeling operating speed," Synthesis Report The National Academies Press, Washington, DC, USA, 2011.

[2] K. Fitzpatrick, O. Wooldridge, J. M. Collins et al., Alternative Design Consistency Rating Methods for Two-Lane Rural Highways, Publication FHWA-RD-99-172, Federal Highway Administration, U.S. Deptartment of Transportation, McLean, VA, USA, 2000.

[3] Transportation Research Board (TRB), "Managing speed: review of current practice for setting and enforcing speed limits," Special Report 254, Austroads, Washington, DC, USA, 1998.

[4] B. Fildes, J. Langford, D. Andrea, and J. Scully, "Balance between harm reduction and mobility in setting speed limits: a feasibility study," Report AP-R272/05, Sydney, Australia, Austroads, 2005.

[5] National Cooperative Highway Research Program (NCHRP), "An Expert System for Recommending Speed Limits in Speed Zones," Project 3-67-Final Report, Transportation Research Board, Washington, DC, USA, 2006.

[6] G. H. D. A. Correia and A. B. Silva, "Setting speed limits on rural two-lane highways by modeling the relationship between expert judgment and measurable roadside characteristics," Journal of Transportation Engineering, vol. 137, no. 3, pp. 184-192, 2010.

[7] A. Lobo, C. Rodrigues, and A. Couto, "Estimating percentile speeds from maximum operating speed frontier," Transportation Research Record, vol. 2404, pp. 1-8, 2014.

[8] A. Lobo, A. Couto, and C. Rodrigues, "Flexible stochastic frontier approach to predict spot speed in two-lane highways," Journal of Transportation Engineering, vol. 142, no. 8, p. 04016032 , 2016.

[9] K. M. Lum, H. S. L. Fan, S. H. Lam, and P. Olszewski, "Speedflow modeling of arterial roads in Singapore," Journal of Transportation Engineering, vol. 124, no. 3, pp. 213-222, 1998.

[10] F. Dion and H. Rakha, "Estimating dynamic roadway travel times using automatic vehicle identification data for low sampling rates," Transportation Research Part B: Methodological, vol. 40, no. 9, pp. 745-766, 2006.
[11] R. Li, G. Rose, and M. Sarvi, "Evaluation of speed-based travel time estimation models," Journal of Transportation Engineering, vol. 132, no. 7, pp. 540-547, 2006.

[12] M. L. Tam and W. H. K. Lam, "Using automatic vehicle identification data for travel time estimation in Hong Kong," Transportmetrica, vol. 4, no. 3, pp. 179-194, 2008.

[13] E. Jenelius and H. N. Koutsopoulos, "Travel time estimation for urban road networks using low frequency probe vehicle data," Transportation Research Part B: Methodological, vol. 53, pp. 6481, 2013.

[14] X. Wang, H. Liu, R. Yu, B. Deng, X. Chen, and B. Wu, "Exploring operating speeds on urban arterials using floating car data: Case study in Shanghai," Journal of Transportation Engineering, vol. 140, no. 9, Article ID 04014044, 2014.

[15] H. Rakha and W. Zhang, "Estimating traffic stream space mean speed and reliability from dual- and single-loop detectors," Transportation Research Record, no. 1925, pp. 38-47, 2005.

[16] F. Soriguera and F. Robusté, "Estimation of traffic stream space mean speed from time aggregations of double loop detector data," Transportation Research Part C: Emerging Technologies, vol. 19, no. 1, pp. 115-129, 2011.

[17] M. Martínez-Díaz and I. Pérez, "A simple algorithm for the estimation of road traffic space mean speeds from data available to most management centres," Transportation Research Part B: Methodological, vol. 75, pp. 19-35, 2015.

[18] D. Park, L. R. Rilett, and G. Han, "Spectral basis neural networks for real-time travel time forecasting," Journal of Transportation Engineering, vol. 125, no. 6, pp. 515-523, 1999.

[19] J. W. C. van Lint, S. P. Hoogendoorn, and H. J. van Zuylen, "Accurate freeway travel time prediction with state-space neural networks under missing data," Transportation Research Part C: Emerging Technologies, vol. 13, no. 5-6, pp. 347-369, 2005.

[20] J. J. Tang, F. Liu, Y. J. Zou, W. B. Zhang, and Y. H. Wang, "An improved fuzzy neural network for traffic speed prediction considering periodic characteristic," IEEE Transactions on Intelligent Transportation Systems, vol. 18, no. 9, pp. 2340-2350, 2017.

[21] Highways Agency (HA), Design Manual for Roads and Bridges, vol. 6, section 1, London, UK, 2002.

[22] Forschungsgesellschaft fuer Strassen-und Verkehrswesen (FGSV), Richtlinien fuer die Anlage von Strassen Strassen-und Verkehrswesen, Cologne, Germany, 2012.

[23] American Association of State Highway and Transportation Officials (AASHTO), A Policy on Geometric Design of Highways and Streets, Washington, DC, USA, 6th edition, 2011.

[24] Junta Autónoma de Estradas (JAE), Norma de Traçado, Almada, Portugal, 1994.

[25] Transportation Research Board (TRB), Highway Capacity Manual 2010, Washington, DC, USA, 2010.

[26] A. Lobo, C. Rodrigues, and A. Couto, "Free-flow speed model based on portuguese roadway design features for two-lane highways," Transportation Research Record, no. 2348, pp. 12-18, 2013.

[27] J. O. Costa, Desenvolvimento dum modelo de previsto de acidentes [Ph.D. Thesis], University of Minho, 2013 (Portuguese).

[28] R. Lamm and E. M. Choueiri, "Recommendation for evaluating horizontal design consistency based on investigations in the state of new york," Transportation Research Record, vol. 1122, pp. 68-78, 1987.

[29] D. R. Jessen, K. S. Schurr, P. T. McCoy, G. Pesti, and R. R. Huff, "Operating speed prediction on crest vertical curves of rural two-lane highways in Nebraska," Transportation Research Record, no. 1751, pp. 67-75, 2001. 
[30] K. S. Schurr, P. T. McCoy, G. Pesti, and R. Huff, "Relationship of design, operating, and posted speeds on horizontal curves of rural two-lane highways in Nebraska," Transportation Research Record, no. 1796, pp. 60-71, 2002.

[31] J. P. Tarris, C. M. Poe, J. M. Mason Jr., and K. G. Goulias, "Predicting operating speeds on low-speed urban streets: regression and panel analysis approaches," Transportation Research Record, no. 1523 , pp. 46-54, 1996.

[32] A. M. F. Medina and A. P. Tarko, "Speed factors on twolane rural highways in free-flow conditions," Transportation Research Record, no. 1912, pp. 39-46, 2005.

[33] P. Hewson, "Quantile regression provides a fuller analysis of speed data," Accident Analysis \& Prevention, vol. 40, no. 2, pp. 502-510, 2008.

[34] D. Aigner, C. A. Lovell, and P. Schmidt, "Formulation and estimation of stochastic frontier production function models," Journal of Econometrics, vol. 6, no. 1, pp. 21-37, 1977.

[35] W. Meeusen and J. van Den Broeck, "Efficiency estimation from cobb-douglas production functions with composed error," International Economic Review, vol. 18, no. 2, pp. 435-444, 1977.

[36] W. H. Greene, Econometric Analysis, Prentice Hall, Upper Saddle River, NJ, USA, 6th edition, 2008.

[37] W. H. Greene, Limdep Version 9.0: Econometric Modeling Guide, Econometric Software, Plainview, NY, USA, 2007. 


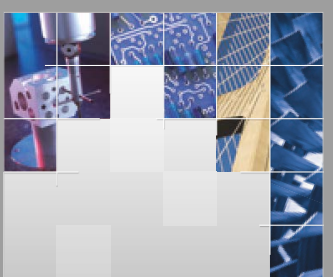

\section{Enfincering}
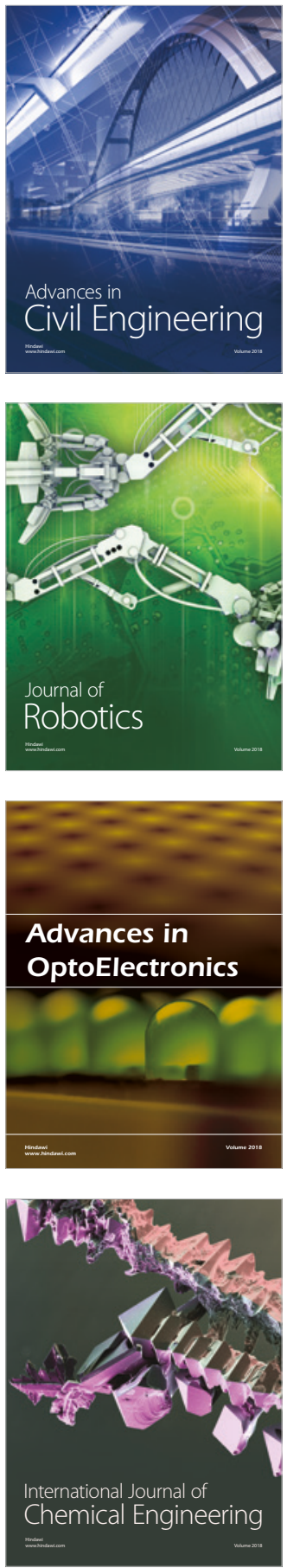

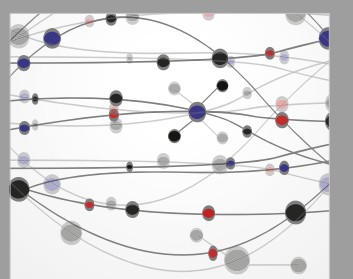

\section{Rotating \\ Machinery}

The Scientific World Journal

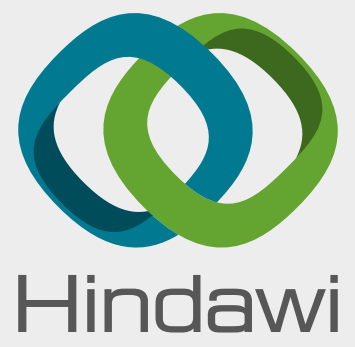

Submit your manuscripts at

www.hindawi.com
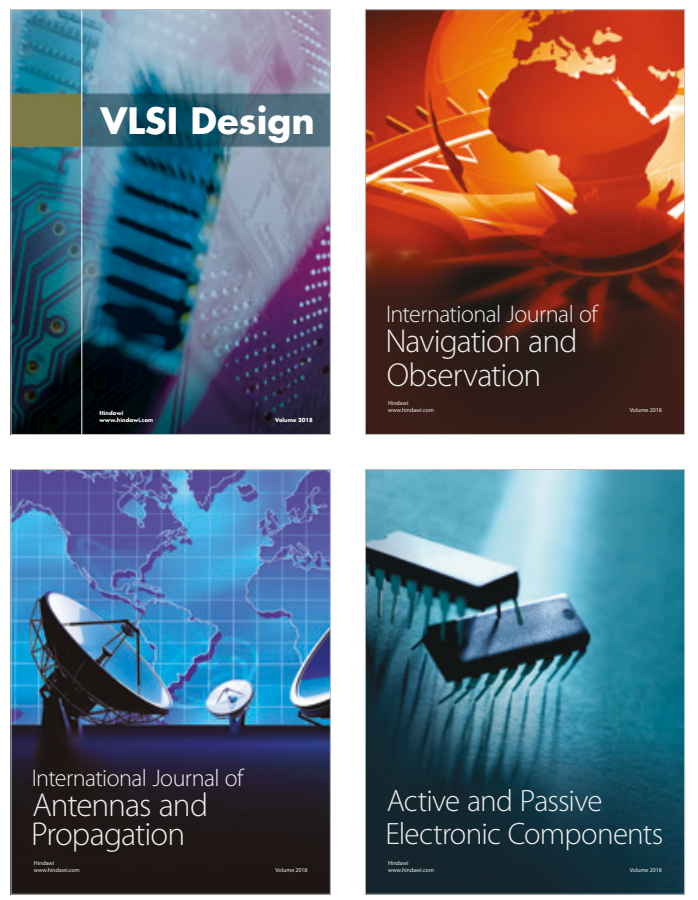
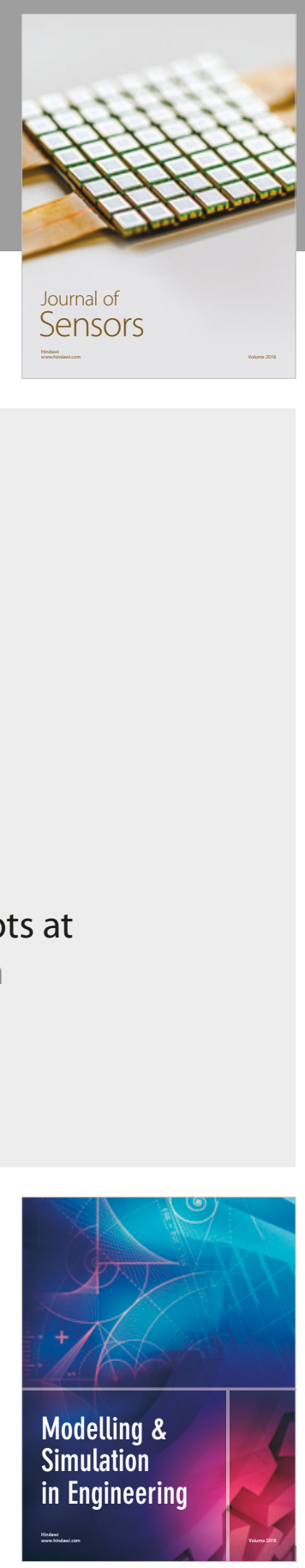

\section{Advances \\ Multimedia}
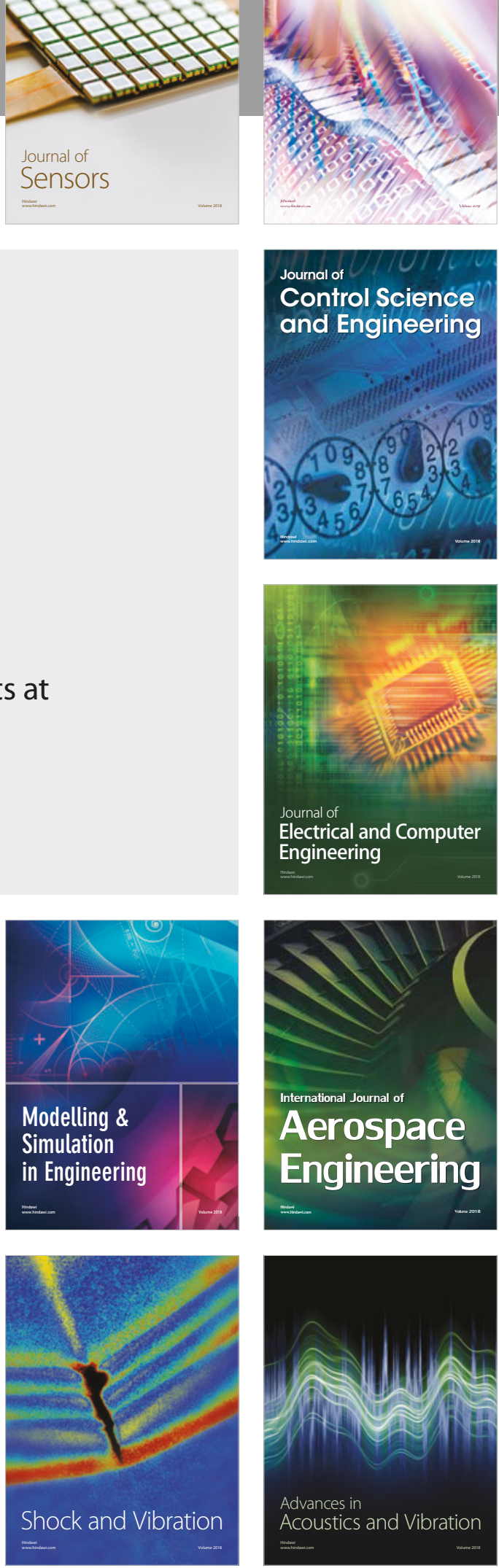